\title{
Roberto Sani, "Unum ovile et unus pastor" ». La compagnia di Gesù e l'esperienza missionaria di padre Matteo Ricci in Cina tra "reformatio Ecclesiae" e inculturazione del Vangelo
}

Rome, Armando Editore, 2010, 147 p.

\section{Michel Ostenc}

\section{OpenEdition}

Journals

\section{Édition électronique}

URL : https://journals.openedition.org/assr/22948

DOI : $10.4000 /$ assr.22948

ISSN : $1777-5825$

Éditeur

Éditions de l'EHESS

\section{Édition imprimée}

Date de publication : 31 décembre 2011

Pagination : 272

ISBN : 9782713223273

ISSN : 0335-5985

\section{Référence électronique}

Michel Ostenc, «Roberto Sani, "Unum ovile et unus pastor" ». La compagnia di Gesù e l'esperienza missionaria di padre Matteo Ricci in Cina tra "reformatio Ecclesiae" e inculturazione del Vangelo ", Archives de sciences sociales des religions [En ligne], 156 | octobre-décembre 2011, document 156-102, mis en ligne le 17 février 2012, consulté le 21 septembre 2021. URL : http://journals.openedition.org/ assr/22948; DOI : https://doi.org/10.4000/assr.22948

Ce document a été généré automatiquement le 21 septembre 2021

(c) Archives de sciences sociales des religions 


\section{Roberto Sani, "Unum ovile et unus pastor" ". La compagnia di Gesù e l'esperienza missionaria di padre Matteo Ricci in Cina tra "reformatio Ecclesiae" e inculturazione del Vangelo}

Rome, Armando Editore, 2010, 147 p.

\section{Michel Ostenc}

\section{RÉFÉRENCE}

Roberto SANI, "Unum ovile et unus pastor" ". La compagnia di Gesù e l'esperienza missionaria di padre Matteo Ricci in Cina tra "reformatio Ecclesiae" e inculturazione del Vangelo, Rome, Armando Editore, 2010, 147 p.

1 La commémoration du $\mathrm{IV}^{\mathrm{e}}$ centenaire de la mort de Matteo Ricci a donné lieu, en mars 2010, à un colloque international à l'université de Macerata, la cité des Marches italiennes dont le père jésuite était originaire. À cette occasion, Roberto Sani a consacré d'importantes études à l'évangélisation de la Chine par le missionnaire en la situant dans le contexte général de l'évolution du catholicisme en Europe aux $\mathrm{XVI}^{\mathrm{e}}$ et $\mathrm{XVII}^{\mathrm{e}}$ siècles. L'auteur démontre, à l'aide d'une abondante documentation puisée notamment dans les Commentaires de Matteo Ricci, publiés entre 1942 et 1949, que l'évangélisation si prisée par l'humanisme de la Renaissance s'inscrivait dans le cadre d'un nouvel universalisme de l'Église post-tridentine. La Compagnie de Jésus en était le protagoniste et elle s'en est fait l'interprète. La statégie missionnaire appliquée aux Indes orientales fut très différente de celle utilisée en Amérique espagnole. Elle 
entendait enraciner le christianisme dans la culture et dans les traditions locales, le père Ricci appliquant cette méthode en Chine dans un processus original d'adaptation de l'Évangile.

2 L'humanisme de la Renaissance s'efforçait de créer entre Dieu et les hommes un équilibre compatible avec la foi et avec la raison qui s'épanouissait dans une spiritualité inspirée de la théologie de Thomas d'Aquin. L'éducation devait par ailleurs retrouver tout son sens et sa dignité première en formant l'homme idéal de son temps. Pendant que les collèges d'enseignement se multipliaient, les Jésuites élaboraient patiemment un ratio studiorum destiné à la formation des élites de l'Europe de la Contre-Réforme et à celles d'un monde nouveau conquis à la foi chrétienne. La lente élaboration de ce cursus s'inscrivait dans une perspective de retour au patrimoine scientifique et littéraire des humanités classiques dont on entendait saisir l'esprit en ne se contentant plus d'une méthode scolastique limitée à la forme ; mais Roberto Sani souligne aussi le caractère individuel de cette formation, chaque individu étant traité en fonction de sa nature. Cette démarche pédagogique était conforme aux prescriptions du concile de Trente qui préconisaient l'établissement de modèles pluralistes valables pour l'Europe, mais aussi pour les terres récemment découvertes. L'auteur estime que les jésuites ont voulu transférer leur méthode scolaire sur le plan de la pastorale afin d'adapter le message chrétien aux peuples évangélisés. Dès l'époque de l'élection de Diego Lainz à la succession d'Ignace de Loyola (1558), un mémoire définissait la nouvelle stratégie missionnaire de l'Ordre de Jésus. L'idée essentielle voulait que la nature humaine fût fondamentalement orientée vers Dieu, dans une vision optimiste d'élévation permanente vers l'idéal de perfection en opposition au pessimisme protestant. Cette conception posait le problème de la grâce, mais soulignait la responsabilité de l'âme dans la construction de son salut. La relation entre l'intervention divine et la liberté de l'homme s'imposait comme une caractéristique des activités pastorales et apostoliques de la Compagnie de Jésus.

3 François-Xavier a été le premier missionnaire jésuite à s'adapter parfaitement aux circonstances et aux conditions locales. Son art de la charité épousait la mentalité de celui qu'il voulait gagner à Dieu, qu'il fût soldat, marchand, artisan ou esclave. Il est certain que les méthodes missionnaires de l'Apôtre des Indes employées à l'égard de populations aux cultures différentes eurent un grand retentissement en Europe et elles décidèrent d'un nombre important de vocations. Le supérieur général de la Compagnie de Jésus Claudio Acquaviva notait, en 1583, cette renovatio spiritus qui s'inspirait de l'action des missionnaires. En 1588, le jésuite Jose de Acosta avait proposé dans son De Natura Novi Orbis une classification des civilisations non chrétiennes. Celles de la Chine et du Japon venaient en premier lieu parce qu'elles étaient jugées plus humaines et politiques, avec un État constitué, des tribunaux, des commerces, des académies et la pratique des lettres. Les populations «barbares» du Nouveau Monde disposaient d'un État organisé, mais elles ignoraient l'étude des lettres, et les peuples sauvages ne possédaient aucune de ces caractéristiques. Ce classement montrait clairement l'intérêt porté à la Chine et au Japon, révélant ainsi les ambitions évangélisatrices de la Compagnie de Jésus dans cette partie du monde. L'inspecteur des missions jésuites du Cap de Bonne-Espérance, Alessandro Valignano, qui se rendit au Japon entre 1578 et 1582, insistait sur la nécessité de concilier l'autorité et la familiarité dans la nouvelle stratégie missionnaire de la Compagnie. Sa correspondance avec Claudio Acquaviva montre que le supérieur général de l'Ordre acceptait cette conception de 
l'évangélisation, tout en émettant des réserves sur l'adoption du mode de vie et de la hiérarchie des moines bouddhistes par les missionnaires jésuites. Enfin, Antonio Possevino, dans son ouvrage d'érudition Biblioteca selecta, ne se limitait pas à l'énoncé de ses controverses avec les protestants, nées de ses tournées apostoliques en Suède et en Europe centrale : il tenait compte de l'expérience de Matteo Ricci et des jésuites en Chine pour définir les caractéristiques du catholicisme de ces missions.

Quand Matteo Ricci arriva en Chine (1583), le message chrétien avait déjà touché le pays à travers des communautés nestoriennes et franciscaines. Le missionnaire apprit le chinois mandarin à Macao, puis à Pékin où l'empereur lui fit une position officielle de mathématicien et d'astronome. À sa mort, le souverain lui concéda une tombe, ce geste passant pour une sorte de reconnaissance légale des jésuites dans l'empire. Dans sa requête au haito de Canton, Matteo Ricci écrivait que les pères jésuites étaient des religieux désireux de demeurer et de mourir en Chine. Roberto Sani montre que la stratégie d'adaptation aux coutumes locales pratiquée par Ricci dans l'Empire céleste s'inspirait de Valignano en matière de spiritualité et de théologie. Ricci voulut évangéliser les mandarins qui formaient l'élite du pays et constituaient l'ossature d'une bureaucratie à laquelle on accédait par des concours difficiles. Savant humaniste affamé de savoir et respectueux de toute connaissance, versé dans les sciences exactes comme dans les humanités classiques, Ricci incarnait parfaitement la culture occidentale de son époque et il se présenta comme tel aux mandarins chinois. Lettré au milieu des lettrés (son nom chinois était Li Matteo), il fut particulièrement apprécié pour ses connaissances en mathématiques et en astronomie. Ricci accordait une grande importance au dialogue afin d'aider l'évangélisation. Pour gagner l'estime de ses hôtes, il inventa des horloges sonnantes et des instruments de musique, et il obtint un grand succès avec sa mappemonde de 1584. Ricci étudia les classiques chinois en insistant sur la conformité de la loi de Dieu avec les enseignements des anciens sages dont la religion chrétienne lui paraissait le couronnement. Il a laissé un livre de philosophie morale, Tien-tchou-chen-i, devenu un classique de la littérature chinoise. Ricci considérait Confucius comme un autre Sénèque et il intégra ses textes dans une vision chrétienne du monde (Les Quatre Livres) pour en faire un nouveau catéchisme (Tien Zhu Shi yi). Ce livre fut très apprécié et décida sans doute l'empereur Kang Hi à promulguer l'édit de 1692 en faveur du christianisme. Il semble que les lettrés n'accordaient aux rites traditionnels qu'une valeur civile et politique, alors que le peuple chinois en faisait un acte religieux. Ricci estima qu'ils ne présentaient pas de caractère idolâtre et il autorisa les convertis à les pratiquer en espérant qu'ils se christianiseraient progressivement.

Roberto Sani estime qu'en s'adressant aux lettrés chinois, Ricci se conforma à la stratégie adoptée par la Compagnie de Jésus pour l'éducation des élites européennes. La nouvelle rhétorique religieuse d'imprégnation humaniste, élaborée pour déterminer le curriculum studiorum des collèges d'enseignement secondaire, a été étendue à d'autres activités des jésuites, en particulier à leurs prêches et à leurs missions. L'auteur s'interroge inversement sur l'influence exercée par l'expérience des missions sur la pédagogie du ratio studiorum qui régla l'organisation des collèges de Jésuites après son adoption, en 1599. Héritiers de l'optimisme humaniste et de sa confiance absolue dans la nature humaine, les jésuites croyaient à une révélation primitive dont les peuples païens conservaient des éléments. Ils apercevaient des similitudes entre l'Ancien Testament et les croyances chinoises. La grâce qui convertit passait par des moyens adaptés à chaque peuple. Les jésuites furent à l'origine de cette insertion des modèles culturels de la tradition païenne dans les principes du christianisme et la participation 
des convertis chinois au culte de leurs ancêtres fut au centre d'une querelle des rites qui devait se prolonger pendant des siècles. 